\section{The heritage of cells}

\begin{tabular}{|l|l} 
& Epigenetics and Chromatin \\
& Edited by Philippe Jeanteur \\
$\begin{array}{l}\text { Epigenetics } \\
\text { and Chromatin }\end{array}$ & Springer-Verlag GmbH $\bullet 2005$ \\
& $\$ 149 / £ 107.50$ \\
& Frank Sauer
\end{tabular}

Metazoan cells can be genetically identical but functionally and structurally distinct. Specific cellular identity is established by cell-type-specific gene expression programmes during development. These expression patterns have to be maintained throughout the life cycle of an organism and, in some cases, they are even transmitted to the offspring. Specialized mechanisms, termed epigenetics, transmit gene expression patterns from one cell generation to the next. Most known epigenetic mechanisms control chromosome activity through post-translational modification of DNA or by dynamic changes in chromatin, a specialized structure composed of histone proteins and DNA.

In the past decade, there have been remarkable advances in our understanding of epigenetic phenomena and the contribution of histone modifications. Given the rapid growth of this area, a book that provides comprehensive coverage of recent advances is needed. Epigenetics and Chromatin now fulfils this need. Edited by Philippe Jeanteur, this book contains ten excellent chapters authored by various researchers in both fields.

A hallmark of epigenetics is the heritable chemical modification of DNA and histones; for example, DNA methylation and histone acetylation and methylation, which mediate mitotic and meiotic propagation of specific expression patterns. These modification patterns have to be maintained during DNA replication, and we know of several molecules that have pivotal roles in these processes. Chapter one introduces the factors involved in DNA and chromatin duplication and describes possible mechanisms for how epigenetic marks are propagated during DNA replication. Chromosomes undergo dramatic structural and functional changes during meiosis. Chapter three describes the cascade of events that culminates in the replacement of histones by protamines and the formation of highly condensed chromatin in spermatozoa. Recent studies that indicate that loci regulated by epigenetic mechanisms remain associated with histones in spermatozoa, and that provide new insights into how epigenetic marks are transmitted from one generation to the next are also discussed.

One of the most exciting revelations to emerge in the epigenetics field is that members of the trithorax group $(\operatorname{Tr} X-G)$ and polycomb group

Frank Sauer is in the Department of Biochemistry, Boyce Hall, University of California Riverside, Riverside 92521, USA.

e-mail:frank.sauer@ucr.edu
(PcG) of epigenetic regulators methylate histones and recruit enzymes that mediate other histone modifications. TrX-G proteins establish and maintain transcriptionally active states, whereas $\mathrm{PcG}$ proteins mediate silencing. Histone modification by these epigenetic regulators not only provided a direct functional link between chromatin and epigenetics but, in addition, indicated that histone modifications can represent epigenetic marks. A discussion of these findings and the function of epigenetic regulators form the basis of Chapter two.

Two chapters provide detailed information on the epigenetic mechanism involving non-coding RNAs and proteins that lead to global hyperactivation and inactivation of $\mathrm{X}$ chromosomes in flies and mammals, respectively.

The importance of DNA methylation - which has an essential role in epigenetic regulation in mammals - and several other model organisms are discussed in Chapters six and seven. These chapters also discuss the role of DNA methylation in development and disease. Additionally, exciting new studies linking DNA methylation with histone modifications, and suggesting that an intricate interplay between DNA and histone modifications controls epigenetic phenomena are also highlighted.

Does epigenetics involve more than histone modification and DNA methylation? To answer this question, it will first be necessary to unravel all the molecular and environmental parameters that direct epigenetic gene expression. Although this task has not yet been achieved, valuable insights into the complexity of epigenetics have been obtained from the detailed analysis of specific gene loci. Chapter eight provides an excellent description of the complexities of epigenetic control by focusing on regulation of the $\beta$-globin locus. The reader will obtain a detailed description of the intricate interplay of specialized DNA elements, higher order chromatin structures and nuclear localization of gene loci in epigenetics.

Imprinting, or the selective inactivation of paternal genes in the zygote, is a special form of epigenetic inheritance. This is not a trivial task, as the zygote has to recognize and inactivate the paternal alleles, while at the same time preserving the function of the maternal copy. Chapter nine provides a compact yet detailed overview of current knowledge and future challenges in this field. Imprinting is not limited to mammals and has been described in insects and plants. An exceptionally well written and accessible comparison of the similarities and differences between parental imprinting in mammals and plants is found in Chapter ten.

Chromatin and Epigenetics thoroughly covers a rapidly developing and expanding field. The different chapters are well written and provide clear, concise and informative views on this topic. Notably, each chapter ends with a conclusion describing current models and future challenges in epigenetics. Although care has been taken to avoid overlap between chapters, in some parts this is unavoidable due to the significant similarities in basic mechanisms that direct epigenetic programmes. Chromatin and Epigenetics covers many important areas in epigenetics, but not all. In particular, chapters describing the role of mammalian epigenetic regulators in development and disease, and the functional connection between RNA interference and epigenetics have not been included.

In summary, both the expert and the novice will find this book a valuable source of information on the various epigenetic phenomena. 